\title{
Reversible splenial lesion and complex visual disturbances due to carbamazepine withdrawal
}

\author{
Rosa Cortese · Giuseppe Pontrelli • Maria Paola Mogavero • \\ Franca Dicuonzo $\cdot$ Carla Tortorella
}

Received: 19 January 2015 / Accepted: 2 March 2015/Published online: 13 March 2015

(C) Springer-Verlag Italia 2015

\section{Dear Sir,}

Reversible, transient and often silent lesions involving the splenium of the corpus callosum (SCC) have been rarely reported in a variety of disorders, including infection, antiepileptic drugs use or withdrawal, metabolic disturbances, and other common demyelinating diseases [1].

Complex visual disturbances may be related to lesions involving the SCC since interhemispheric fibers linking the activated visual areas, pass through the SCC and integrate visual information as demonstrated by electrophysiological, neuroanatomical and functional magnetic resonance imaging findings $[2,3]$.

We report the case of a young patient showing a reversible, Gadolinium enhancing SCC lesion associated with visual disturbances after carbamazepine withdrawal.

A 24-year-old girl was admitted to our hospital, complaining about acute balance loss and altered visual perception. She described a persistent perception of quadruple images on binocular vision. She had a history of episodic migraine without aura. One month before hospital

\author{
R. Cortese - G. Pontrelli - M. P. Mogavero - F. Dicuonzo · \\ C. Tortorella $(\square)$ \\ Department of Basic Medical Sciences, Neurosciences \\ and Sense Organs, University of Bari, Piazza G. Cesare, 11, \\ 70124 Bari, Italy \\ e-mail: carla.tortorella@gmail.com \\ R. Cortese \\ e-mail: rosanna.cortese@gmail.com \\ G. Pontrelli \\ e-mail: giumus21@ hotmail.com \\ M. P. Mogavero \\ e-mail: paola_mogavero@libero.it \\ F. Dicuonzo \\ e-mail: franca.dicuonzo@uniba.it
}

admission, because of trigeminal neuralgia, she was treated with carbamazepine $400 \mathrm{mg}$ /day, abruptly interrupted after 15 days. No other systemic diseases or family history of neurological diseases were reported. Neurological and ophthalmologic evaluations were normal except for reduced visual acuity (3/10 bilaterally). She had no cognitive deficit, nor psychiatric disturbances. Standard blood tests revealed no abnormalities. Antinuclear, antimicrosomal and antithyroglobulin antibodies were negative. Cerebrospinal fluid (CSF) showed normal cell count and protein content, as well as no IgG intrathecal synthesis. Serum and CSF test for Treponema pallidum and Lyme, PCR tests for virus and anti-AQP4 antibodies were negative. Brain MRI scans showed a single T2-hyperintense, gadolinium enhancing lesion of the splenium of the corpus callosum (Fig. 1a, b). No spinal cord MRI lesions were found. Visual, brainstem auditory and somatosensory evoked potentials were normal and no abnormalities were demonstrated by computerized visual field. After 5 gr intravenous methylprednisolone therapy visual acuity improved (8/10) bilaterally and "quadruple vision" resolved. One month follow-up MRI showed a quite complete remission of the SCC lesion (Fig. 2a, b). MRI was completely normal after 6 months. Clinical and MRI evaluation after 2 years showed no abnormalities.

The possibility of symptomatic, reversible SCC lesions due to reversible demyelination or cytotoxic edema following sudden withdrawal of carbamazepine assumption must be taken into account in clinical practice. The transient impairment of visual acuity in spite of normal visual evoked potentials and computerized visual field suggests impairment related to patient compliance and binocular dysfunction more than to abnormalities of the visual pathways [4]. Visual acuity improvement after the resolution of the "quadruple vision" confirms such hypothesis 
Fig. 1 Baseline MRI scans. T2weighted scan showed hyperintense SCC lesion (a), slightly enhancing after gadolinium administration $(\mathbf{b})$
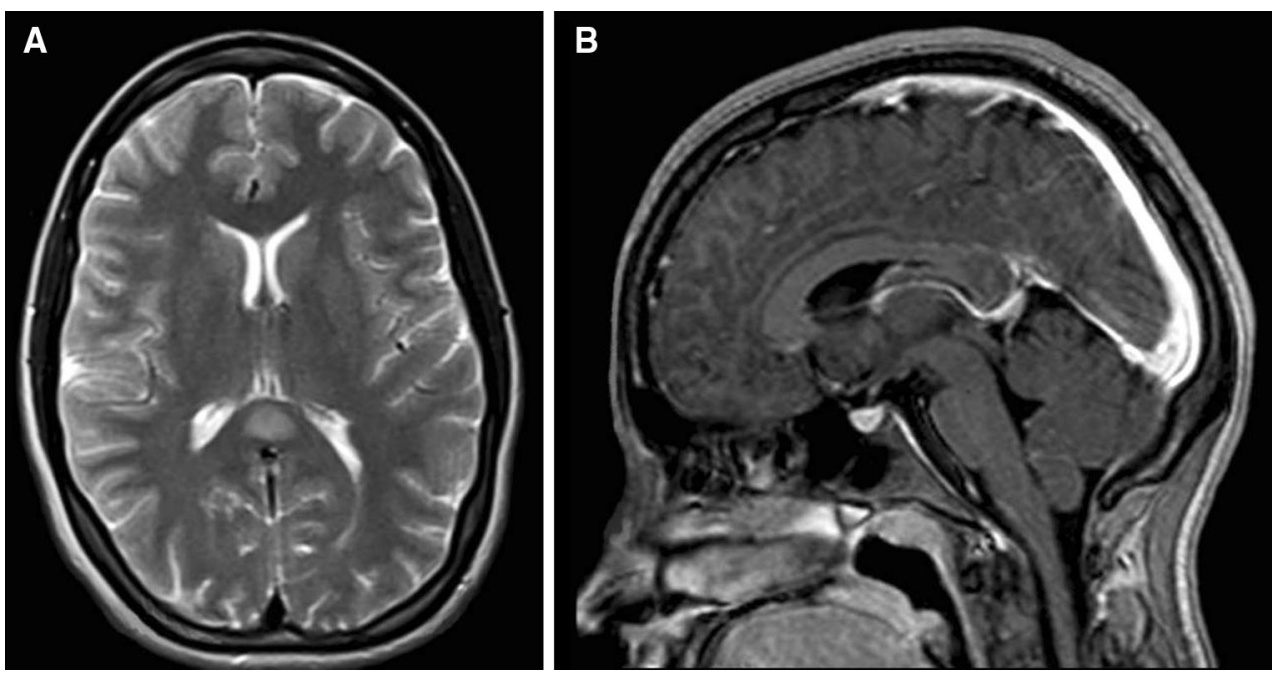
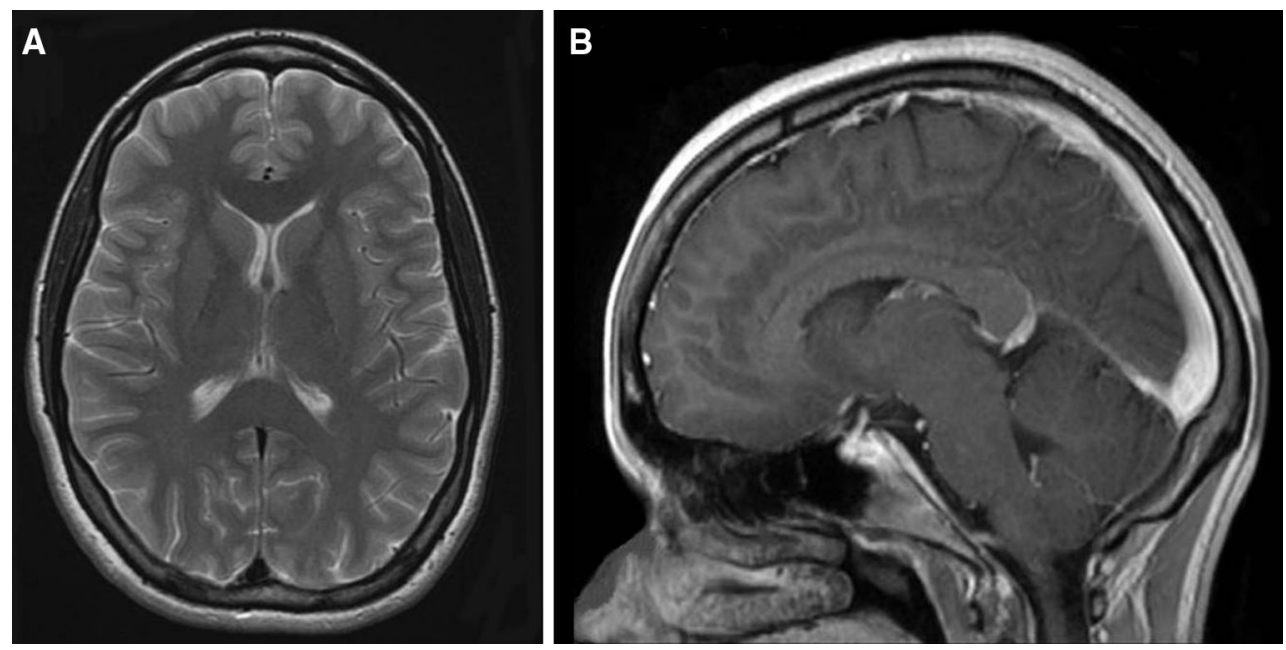

Fig. 2 Follow-up MRI scans. One month after symptoms onset the SCC lesion disappeared on both T2-weighted (a) and T1-weighted (b) MR images

and that the importance of SCC for the interhemispheric interaction between the cortical regions goes well beyond the visual modality [5].

Conflict of interest Dr. Tortorella received honoraria for speaking from Biogen-Idec, Sanofi-Aventis, Merck Serono, Novartis, BayerSchering, and Teva Pharmaceutical. No disclosures were reported by other authors.

\section{References}

1. Garcia-Monco JC, Cortina IE, Ferreira E, Martínez A, Ruiz L, Cabrera A, Beldarrain MG (2011) Reversible splenial lesion syndrome (RESLES): what's in a name? J Neuroimaging 21:e1e14

2. Pietrasanta M, Restani L, Caleo M (2012) The corpus callosum and the visual cortex: plasticity is a game for two. Neural Plast 2012:838672. doi:10.1155/2012/838672 (1-10)

3. Winslow H, Mickey B, Frohman EM (2006) Sympathomimeticinduced kaleidoscopic visual illusion associated with a reversible splenium lesion. Arch Neurol 63:135-137

4. Denniston AK, Dick AD (2013) Systemic therapies for inflammatory eye disease: past, present and future. BMC Ophthalmol 24(13):18. doi:10.1186/1471-2415-13-18

5. Berlucchi G (2014) Visual interhemispheric communication and callosal connections of the occipital lobes. Cortex 56:1-13. doi:10. 1016/j.cortex.2013.02.001 